\title{
INLUENCE OF DIFFERENT LEVELS OF COWDUNG ON MITIGATION OF WATER DEFICIT EFFECT ON WHEAT
}

\author{
A.K.M.R. Amin ${ }^{1}$ and S. Reza ${ }^{2}$ \\ ${ }^{1}$ Professor and ${ }^{2} \mathrm{MS}$ Student, Department of Agronomy, Sher-e-Bangla Agricultural University \\ Corresponding E-mail: ruhulsau@yahoo.com
}

(Received: 10 April 2021, Accepted: 20 April 2021)

Keywords: Organic manure, water deficit, growth stages, yield, wheat

\begin{abstract}
The experiment was conducted in pot at the net house of the department of Agronomy, Sher-e-Bangla Agricultural University, Dhaka during the period from November, 2018 to March, 2019to find out the optimum dose(s) cowdung to mitigate the water deficit effect on wheat. The experiment comprised of two factors viz. factor A: Five levels of cowdung, i) $C_{0}=$ Control (No cowdung), $C_{1}=25 \%$ less cowdung of recommended dose, $\mathrm{C}_{2}=$ Recommended dose of cowdung, $\mathrm{C}_{3}=25 \%$ higher cowdung of recommended dose and $\mathrm{C}_{4}=50 \%$ higher cowdung of recommended dose, and factor $\mathrm{B}$ : four levels of water deficit at, i) $\mathrm{D}_{0}=$ Control (No water deficit), $\mathrm{D}_{1}=$ Crown root initiation stage (20-19 DAS), $\mathrm{D}_{2}=$ Booting stage (45-54 DAS) and $\mathrm{D}_{3}=\mathrm{An}$ thesis stage (55-64 DAS). The experiment was laid out in a Factorial $\mathrm{R}$ and omized Complete Block Design with three replications. The test crop variety was BARI Gom28. The result reveled that cowdung level had positive impact on yield of wheat under water deficit condition, and $50 \%$ higher cowdung of recommended dose $\left(C_{4}\right)$ gave the highest grain yield $\left(5.12 \mathrm{~g}\right.$ plant $\left.{ }^{-1}\right)$. The particular treatment also produced the highest number of effective tillers plant ${ }^{-1}$ (5.25), spike length $(10.39 \mathrm{~cm})$, spikelet spike ${ }^{-1}(15.72)$, grains spike ${ }^{-1}(32.56)$, grains spikelet ${ }^{-1}$ (2.07) and 1000 -grain weight $(47.32 \mathrm{~g})$ of wheat. The treatment $\mathrm{C}_{3}(25 \%$ higher cowdung of recommended dose) also gave statistically similar yield with $\mathrm{C}_{4}$ treatment. In respect of water deficit imposition treatments, grain yield was found the highest in control treatment which was statistically similar with water deficit imposition at booting stage treatment $\left(\mathrm{D}_{2}\right)$. These two treatments also showed the higher and similar number of effective tillers plant ${ }^{-1}$ (4.86 and 4.58), spike length $(10.53 \mathrm{~cm}$ and $10.11 \mathrm{~cm})$, spikelets spike ${ }^{-1}(15.50$ and 15.19$)$, grains spike ${ }^{-1}(34.10$ and 30.17), grains spikelet ${ }^{-1}$ (2.20 and 1.98) and 1000-grain weight (45.42g and $45.36 \mathrm{~g}$, respectively). Regarding the interaction of levels of cowdung and water deficit imposition at different stages of plant growth, $C_{4} D_{0}$ and $C_{3} D_{0}$ were highest yielder which was attributed to higher 1000 -seed weight, number of effective tillers plant $^{-1}$, spikelets spike ${ }^{-1}$ and grains spike ${ }^{-1}$. Contrary, 25\% higher cowdung than recommended dose (as it saved 25\% cowdung) seems promising to overcome yield loss due to water deficit imposition at booting stage of wheat $\left(D_{2}\right)$. However, application of cowdung $\left(12.5 \mathrm{t} \mathrm{ha}^{-1}\right)$ was found effective to combat water deficit at booting stage $\left(\mathrm{D}_{2}\right)$ of wheat compared to other growth stages.
\end{abstract}

\section{Introduction}

Wheat production of Bangladesh was 11.0 lac metric tons and area cover 3.5 lac hectares (BBS, 2019). Drought affects all plant development stages from germination, vegetative and 
reproductive growth to grain filling and maturation of the crop (Hossain et al., 2012). Drought reduces nitrogen $(\mathrm{N})$ uptake efficiency and utilization by plants. Drought is one of the major abiotic stresses that affect at least $60 \%$ of wheat production in high-income countries and about $32 \%$ of 99 million hectares in least developed countries (Chen et al., 2012). Water deficit might decrease wheat grain yield from 17 to 70\% (Nouri-Ganbalani et al., 2009). Daryanto et al. (2016) reported $20.6 \%$ yield losses in $40 \%$ reduced water. Sarwar (2005) found that grain yield and yield components of wheat significantly increased with the application of different organic materials resulting in the compost to be the most superior one. In addition, Yassen et al. (2006) found that the irrigation at $60 \%$ water holding capacity and applying mineral $60 \mathrm{~kg} \mathrm{Nfed}{ }^{-1}$, with presence of the chicken manure as an organic fertilizer produced the highest wheat yield. On the other hand, Amin and Baque (2020) observed that application of organic manure could reduce the impact of drought on wheat irrespective of growth stages. They also observed that application of cowdung (10 t ha-1) was found more effective to combat drought impact at booting stage of wheat. As such, this research work was designed to determine the effect of different levels of cowdung on mitigation of water deficit effect on wheat.

\section{Materials and Methods}

Apot experiment was conducted at the net house of the department of Agronomy, Sher-eBangla Agricultural University (SAU), Dhaka-1207, during the period of November 2018 to March 2019. The experimental field was located at 240. $09^{\prime} \mathrm{N}$ latitude and $90^{\circ} 26^{\prime} \mathrm{E}$ longitude at a height of $8.5 \mathrm{~m}$ above the sea level (FAO/UNDP, 1988). The soil of the experimental site was clay loam belonging to the "Madhupur Tract" under AEZ 28. Two factors experiment werefactor A: Five levels of cowdung, viz.i) $\mathrm{C}_{0}=$ Control (No cowdung), $\mathrm{C}_{1}=25 \%$ less cowdung of recommended dose, $\mathrm{C}_{2}=$ Recommended dose of cowdung, $\mathrm{C}_{3}=25 \%$ higher cowdung of recommended dose and $\mathrm{C}_{4}=50 \%$ higher cowdung of recommended dose, and factor $\mathrm{B}$ : four levels of water deficit at, i) $\mathrm{D}_{0}=$ Control (No water deficit), $\mathrm{D}_{1}=$ Crown root initiation stage (2019 DAS), $\mathrm{D}_{2}=$ Booting stage (45-54 DAS) and $\mathrm{D}_{3}=$ Anthesis stage (55-64 DAS) (Amin and Baque, 2020). The experiment was laid out in a Factorial $\mathrm{R}$ and omized Complete Block Design with three replications. The test crop variety of wheat was BARI Gom28. Sixty earthen pots measuring $22 \mathrm{~cm}$ diameter and $18 \mathrm{~cm}$ height was fill-up with $20 \mathrm{~kg}$ of soil. Urea, TSP, MoP, Gypsum, Zincoxide and Boric acid were used at the rate of 200,72,66, 110, 4 and $5 \mathrm{~kg} \mathrm{ha}^{-1}$, respectively (BRRI, 2006 / FRG, 2018), which were 2.00, 0.72, 0.66, 1.10, 0.04 and $0.05 \mathrm{~g}$ pot $^{-1}$, respectively and mixed all of them except urea with the soil before fill-up the pot. Urea was applied in three equal installments at pot filling, 21 DAS and 55 DAS. Recommended dose of cowdung was $10 \mathrm{t} \mathrm{ha}^{-1}$ and was applied as per treatment. Seeds of wheat variety BARI Gom28 were collected from Bangladesh Agriculture Research Institute (BARI), Joydebpur, Gazipur. Before sowing, seeds were treated with Provex 200EC @ $2.5 \mathrm{~g}$ powder for $\mathrm{kg}^{-1}$ seed. Fifteen seeds were sown in each pot on 21st November 2018. After sowing, the seeds were covered with soil and lightly pressed by hand. For assessment, five plants were kept in each pot after 14 DAS. Different intercultural operations were done to ensure normal growth and development of the crop except irrigation. Irrigation was applied as per need of treatment of the experiment where irrigation was not applied during water deficit imposition period(s) treatments. On the basis of physiological maturity, the crop was harvested from 4-10 March, 2019. Data on different crop characters, yield attributes and yield were collected from the harvested five plants from each pot. Post-harvest operations like- threshing, cleaning and drying of grains were done separately for each treatment. Properly dried grain and straw were weighed and converted into $g$ plant $^{-1}$ basis. The collected data of each pot were statistically analyzed by using the computer- 
based software Statistics 10. Mean difference among the treatments were compared with Duncan's Multiple Range Test (DMRT) test at $5 \%$ level of significance.

\section{Results and Discussion}

The result reveled that different cowdung treatment varied significantly with respect to yield and yield contributing characters of wheat (Table 1 and Table 2). The yield advantages of 0.78 , $0.48,0.38$ and $0.13 \mathrm{~g}$ plant $^{-1}$ for $\mathrm{C}_{4}$ (50\% higher cowdung of recommended dose) applied pot over $\mathrm{C}_{0}$ (No cowdung), $\mathrm{C}_{1}$ (25\% less cowdung of recommended dose), $\mathrm{C}_{2}$ (Recommended dose of cowdung) and $\mathrm{C}_{3}$ (25\% higher cowdung of recommended dose, respectively) applied pot was found possibly due to maximum effective tillers plant ${ }^{-1}(5.25)$, spike length $(10.39 \mathrm{~cm})$, spikelets spike $^{-1}$ (15.72), grains spike ${ }^{-1}$ (32.56), grains spikelet ${ }^{-1}$ (2.07), weight of 1000 grains (47.32 g), straw yield (6.47 $\left.\mathrm{g} \mathrm{plant}^{-1}\right)$, biological yield (11.39 $\left.\mathrm{g} \mathrm{plant}^{-1}\right)$ and harvest index $(44.08 \%)$ in the $\mathrm{C}_{4}$ applied treatment. On the other hand, $\mathrm{C}_{3}$ treatment gave statistically similar yield and yield attributes with $\mathrm{C}_{4}$ treatment in some traits. The result agreed with the findings of Amin and Baque (2020), Hammad et al. (2011) and Ibrahim et al. (2008) that organic manure increased wheat yield over control. According to Uyanoz et al. (2006) yield attributes of wheat improve with organic manure which corroborates with the present results.

Table 1. Effect of different levels of cowdung on plant characters and yield attributes of wheat

\begin{tabular}{lccccccc}
\hline $\begin{array}{c}\text { Cowdung } \\
\text { dose }\end{array}$ & $\begin{array}{c}\text { Plant } \\
\text { height } \\
\text { (cm) }\end{array}$ & $\begin{array}{c}\text { Effective } \\
\text { tillers plant }^{-1} \\
\text { (no.) }\end{array}$ & $\begin{array}{c}\text { Spike } \\
\text { length } \\
\text { (cm) }\end{array}$ & $\begin{array}{c}\text { Spikelets } \\
\text { spike }^{-1} \\
\text { (no.) }\end{array}$ & $\begin{array}{c}\text { Grains }_{\text {spike }^{-1}} \\
\text { (no.) }^{-1}\end{array}$ & $\begin{array}{c}\text { Grains } \\
\text { spikelet }^{-1} \\
\text { (no.) }\end{array}$ & $\begin{array}{c}\text { Weight of } \\
\text { 1000 grains } \\
\text { (g) }\end{array}$ \\
\hline $\mathrm{C}_{0}$ & $66.65 \mathrm{c}$ & $2.94 \mathrm{e}$ & $9.48 \mathrm{c}$ & $14.07 \mathrm{~b}$ & $22.36 \mathrm{~d}$ & $1.58 \mathrm{c}$ & $39.51 \mathrm{c}$ \\
$\mathrm{C}_{1}$ & $68.07 \mathrm{bc}$ & $3.56 \mathrm{~d}$ & $9.68 \mathrm{bc}$ & $14.25 \mathrm{~b}$ & $26.25 \mathrm{c}$ & $1.84 \mathrm{~b}$ & $41.86 \mathrm{~b}$ \\
$\mathrm{C}_{2}$ & $68.08 \mathrm{bc}$ & $3.90 \mathrm{c}$ & $9.82 \mathrm{bc}$ & $14.48 \mathrm{~b}$ & $28.78 \mathrm{~b}$ & $1.99 \mathrm{ab}$ & $42.85 \mathrm{~b}$ \\
$\mathrm{C}_{3}$ & $69.97 \mathrm{ab}$ & $4.68 \mathrm{~b}$ & $10.17 \mathrm{ab}$ & $15.50 \mathrm{a}$ & $31.36 \mathrm{a}$ & $2.02 \mathrm{ab}$ & $46.58 \mathrm{a}$ \\
$\mathrm{C}_{4}$ & $72.14 \mathrm{a}$ & $5.25 \mathrm{a}$ & $10.39 \mathrm{a}$ & $15.72 \mathrm{a}$ & $32.56 \mathrm{a}$ & $2.07 \mathrm{a}$ & $47.32 \mathrm{a}$ \\
\hline $\mathrm{SE}$ & 1.33 & 0.13 & 0.27 & 0.34 & 0.71 & 0.08 & 1.00 \\
$\mathrm{CV}(\%)$ & 4.71 & 8.07 & 6.78 & 5.58 & 6.18 & 10.87 & 5.62 \\
\hline
\end{tabular}

In a column means having similar letter(s) are statistically similar and those having dissimilar letter(s) differ significantly at $5 \%$ level of significance by DMRT.

Here: $\mathrm{C}_{0}=$ Control (No cowdung), $\mathrm{C}_{1}=25 \%$ less cowdung of recommended dose, $\mathrm{C}_{2}=$ Recommended dose of cowdung, $\mathrm{C}_{3}=25 \%$ higher cowdung of recommended dose and $\mathrm{C}_{4}=50 \%$ higher cowdung of recommended dose

Table 2. Effect of different levels of cowdung on yield and harvest index of wheat

\begin{tabular}{ccccc}
\hline Cowdung dose & $\begin{array}{c}\text { Grain yield } \\
\text { plant }^{-1} \\
(\mathbf{g})\end{array}$ & $\begin{array}{c}\text { Straw yield } \\
\text { plant }^{-1} \\
(\mathbf{g})\end{array}$ & $\begin{array}{c}\text { Biological } \\
\text { yield plant }\end{array}$ & $\begin{array}{c}\text { Harvest index } \\
\text { (g) }\end{array}$ \\
\hline $\mathrm{C}_{0}$ & $4.34 \mathrm{~d}$ & $5.90 \mathrm{~b}$ & $10.24 \mathrm{~b}$ & 42.29 \\
$\mathrm{C}_{1}$ & $4.64 \mathrm{c}$ & $6.08 \mathrm{ab}$ & $10.72 \mathrm{~b}$ & 43.21 \\
$\mathrm{C}_{2}$ & $4.76 \mathrm{bc}$ & $6.17 \mathrm{ab}$ & $10.79 \mathrm{~b}$ & 43.47 \\
$\mathrm{C}_{3}$ & $4.99 \mathrm{ab}$ & $6.50 \mathrm{a}$ & $11.48 \mathrm{a}$ & 43.37 \\
$\mathrm{C}_{4}$ & $5.12 \mathrm{a}$ & $6.47 \mathrm{a}$ & $11.59 \mathrm{a}$ & 44.08 \\
\hline $\mathrm{SE}$ & 0.126 & 0.247 & 0.294 & $\mathrm{NS}$ \\
$\mathrm{CV}(\%)$ & 6.45 & 9.72 & 6.56 & 5.84 \\
\hline
\end{tabular}

In a column means having similar letter(s) are statistically similar and those having dissimilar letter(s) differ significantly at $5 \%$ level of significanc by DMRT.

Here: $\mathrm{C}_{0}=$ Control (No cowdung), $\mathrm{C}_{1}=25 \%$ less cowdung of recommended dose, $\mathrm{C}_{2}=$ Recommended dose of cowdung, $\mathrm{C}_{3}=25 \%$ higher cowdung of recommended dose and $\mathrm{C}_{4}=50 \%$ higher cowdung of recommended dose, and NS = Not significant 
Significant difference existed among the water deficit-imposed treatments, the control plants $\mathrm{D}_{0}$ and $\mathrm{D}_{2}$ showed maximum statistically similar grain yield, spike length, spikelets spike ${ }^{-1}$, weight of 1000 grains and harvest index (Table 3 and Table 4). Without drought treatment was superior by producing 34.41 and $19.78 \%$ higher yield over $\mathrm{D}_{3}$ and $\mathrm{D}_{1}$ treatments, respectively. On the other hand, $\mathrm{D}_{2}$ treatment was out yielded by producing 29.18 and $15.11 \%$ higher yield over $\mathrm{D}_{3}$ and $\mathrm{D}_{1}$, respectively. The treatment without drought also produced highest level of tillers plant ${ }^{-1}$, spikelets spike $e^{-1}$, grains spike ${ }^{-1}$, straw yield, biological yield and harvest index than drought imposition plants. However, among the drought imposition treatments, $\mathrm{D}_{2}$ gave highest yield and yield attributes than other drought imposition treatments. The present result was confirmatory with the findings of Amin and Baque (2020) and Akram (2011) that drought imposition at different growth stages caused severe reduction in yield and yield components of wheat. Similar result was also observed by Alghabari and Isham (2018) that drought stress affected barley yield through impaired grain development and grain filling duration.

Table 3. Effect of water deficit treatment on plant characters and yield attributes of wheat

\begin{tabular}{cccccccc}
\hline $\begin{array}{c}\text { Water } \\
\text { deficit } \\
\text { stage }\end{array}$ & $\begin{array}{c}\text { Plant } \\
\text { height } \\
\text { (cm) }\end{array}$ & $\begin{array}{c}\text { Effective } \\
\text { tillers } \\
\text { plant }^{-1}\end{array}$ & $\begin{array}{c}\text { Spike } \\
\text { length } \\
\text { (cm) }\end{array}$ & $\begin{array}{c}\text { Spikelets } \\
\text { spike }^{-1}\end{array}$ & $\begin{array}{c}\text { Grains } \\
\text { spike }^{-1}\end{array}$ & $\begin{array}{c}\text { Grains } \\
\text { spikelet }^{-1}\end{array}$ & $\begin{array}{c}\text { Weight of } \\
\text { 1000 grains } \\
\text { (g) }\end{array}$ \\
\hline $\mathrm{D}_{0}$ & $73.41 \mathrm{a}$ & $4.86 \mathrm{a}$ & $10.53 \mathrm{a}$ & $15.50 \mathrm{a}$ & $34.10 \mathrm{a}$ & $2.20 \mathrm{a}$ & $45.42 \mathrm{a}$ \\
$\mathrm{D}_{1}$ & $70.75 \mathrm{~b}$ & $3.94 \mathrm{c}$ & $9.69 \mathrm{bc}$ & $14.75 \mathrm{~b}$ & $26.08 \mathrm{c}$ & $1.76 \mathrm{c}$ & $42.87 \mathrm{~b}$ \\
$\mathrm{D}_{2}$ & $67.31 \mathrm{c}$ & $4.58 \mathrm{~b}$ & $10.11 \mathrm{ab}$ & $15.19 \mathrm{ab}$ & $30.17 \mathrm{~b}$ & $1.98 \mathrm{~b}$ & $45.36 \mathrm{a}$ \\
$\mathrm{D}_{3}$ & $64.46 \mathrm{~d}$ & $2.88 \mathrm{~d}$ & $9.29 \mathrm{c}$ & $13.84 \mathrm{c}$ & $22.70 \mathrm{~d}$ & $1.62 \mathrm{c}$ & $40.84 \mathrm{c}$ \\
\hline $\mathrm{SE}$ & 1.19 & 0.12 & 0.24 & 0.30 & 0.64 & 0.07 & 0.89 \\
$\mathrm{CV}(\%)$ & 4.71 & 8.07 & 6.78 & 5.58 & 6.18 & 10.87 & 5.62 \\
\hline
\end{tabular}

In a column means having similar letter(s) are statistically similar and those having dissimilar letter(s) differ significantly at $5 \%$ level of significance

Here: $D_{0}=$ Control (No water deficit), $D_{1}=$ Water deficit at crown root initiation stage (20-19 DAS), $D_{2}=$ Water deficit atbooting stage (45-54 DAS) and $\mathrm{D}_{3}=$ Water deficit at anthesis stage (55-64 DAS)

Table 4. Effect of water deficit treatment on yield and harvest index of wheat

\begin{tabular}{ccccc}
\hline $\begin{array}{c}\text { Water deficit } \\
\text { stage }\end{array}$ & $\begin{array}{c}\text { Grain yield } \\
\text { plant }^{-1} \\
(\mathbf{g})\end{array}$ & $\begin{array}{c}\text { Straw yield } \\
\text { plant }^{-1} \\
(\mathbf{g})\end{array}$ & $\begin{array}{c}\text { Biological } \\
\text { yield plant }\end{array}$ & $\begin{array}{c}\text { Harvest index } \\
\text { (g) }\end{array}$ \\
\hline $\mathrm{D}_{0}$ & $5.39 \mathrm{a}$ & $6.72 \mathrm{a}$ & $12.11 \mathrm{a}$ & $44.47 \mathrm{a}$ \\
$\mathrm{D}_{1}$ & $4.50 \mathrm{~b}$ & $6.01 \mathrm{~b}$ & $10.51 \mathrm{~b}$ & $42.82 \mathrm{ab}$ \\
$\mathrm{D}_{2}$ & $5.18 \mathrm{a}$ & $6.63 \mathrm{a}$ & $11.70 \mathrm{a}$ & $43.84 \mathrm{ab}$ \\
$\mathrm{D}_{3}$ & $4.01 \mathrm{c}$ & $5.53 \mathrm{c}$ & $9.54 \mathrm{c}$ & $41.99 \mathrm{~b}$ \\
\hline $\mathrm{SE}$ & 0.11 & 0.23 & 0.26 & 0.92 \\
$\mathrm{CV}(\%)$ & 6.45 & 9.72 & 6.56 & 5.84 \\
\hline
\end{tabular}

In a column means having similar letter(s) are statistically similar and those having dissimilar letter(s) differ significantly at $5 \%$ level of significance.

$\mathrm{D}_{0}=$ Control (No water deficit), $\mathrm{D}_{1}=$ Water deficit at crown root initiation stage (20-19 DAS), $\mathrm{D}_{2}=$ Water deficit atbooting stage (45-54 DAS) and $\mathrm{D}_{3}=$ Water deficit at anthesis stage (55-64 DAS). 
Interaction of cowdung level and water deficit imposition treatments showed significant variation in all the studied parameters (Table 5 and Table 6). The interaction of $\mathrm{C}_{4} \mathrm{D}_{0}$ and $\mathrm{C}_{3} \mathrm{D}_{0}$ performed best in respect of grain yield (5.93 and $5.62 \mathrm{~g} \mathrm{plant}^{-1}$, respectively) which may be attributed to highest effective tillers plant ${ }^{-1}$, spike length, spikelets spike ${ }^{1}$, grains spikelet ${ }^{1}$ and weight of 1000 grains in these interactions. On the other hand, interaction of $\mathrm{C}_{3} \mathrm{D}_{2}$ also showed statistically similar yield $\left(5.48 \mathrm{~g} \mathrm{ha}{ }^{-1}\right)$ and yield contributing characters with $\mathrm{C}_{4} \mathrm{D}_{0}$ and $\mathrm{C}_{3} \mathrm{D}_{0}$ interactions.

Table 5. Interaction effects of different levels of cowdung and water deficit imposition treatment on plant characters and yield attributes of wheat

\begin{tabular}{|c|c|c|c|c|c|c|c|}
\hline Interaction & $\begin{array}{l}\text { Plant } \\
\text { height } \\
(\mathrm{cm})\end{array}$ & $\begin{array}{c}\text { Effective } \\
\text { tillers } \\
\text { plant }^{-1}\end{array}$ & $\begin{array}{l}\text { Spike } \\
\text { length } \\
(\mathrm{cm})\end{array}$ & $\begin{array}{l}\text { Spikelets } \\
\text { spike }^{-1}\end{array}$ & $\begin{array}{l}\text { Grains } \\
\text { spike }^{-1}\end{array}$ & $\begin{array}{l}\text { Grains } \\
\text { spikelet }^{-1}\end{array}$ & $\begin{array}{l}\text { Weight of } \\
\text { 1000- grain } \\
\text { (g) }\end{array}$ \\
\hline $\mathrm{C}_{0} \mathrm{D}_{0}$ & $71.67 \mathrm{a}-\mathrm{d}$ & $3.81 \mathrm{fg}$ & $10.26 \mathrm{a}-\mathrm{g}$ & $14.71 \mathrm{c}-\mathrm{h}$ & $26.99 \mathrm{f}-\mathrm{h}$ & $1.83 e-h$ & 42.63 ef \\
\hline $\mathrm{C}_{0} \mathrm{D}_{1}$ & $68.67 \mathrm{c}-\mathrm{g}$ & $2.22 \mathrm{k}$ & $9.16 \mathrm{~g}-\mathrm{i}$ & $13.73 \mathrm{~g}-\mathrm{i}$ & $21.18 \mathrm{j}$ & $1.54 \mathrm{~h}-\mathrm{i}$ & $38.04 \mathrm{~g}$ \\
\hline $\mathrm{C}_{0} \mathrm{D}_{2}$ & $64.85 \mathrm{gh}$ & $3.43 \mathrm{gh}$ & $9.47 \mathrm{c}-\mathrm{i}$ & $14.42 \mathrm{~d}-\mathrm{i}$ & $24.55 \mathrm{hi}$ & $1.70 \mathrm{gh}$ & $40.35 \mathrm{fg}$ \\
\hline $\mathrm{C}_{0} \mathrm{D}_{3}$ & $61.40 \mathrm{~h}$ & $2.31 \mathrm{jk}$ & $9.01 \mathrm{i}$ & $13.42 \mathrm{hi}$ & $16.72 \mathrm{k}$ & $1.25 \mathrm{i}$ & $37.03 \mathrm{~g}$ \\
\hline $\mathrm{C}_{1} \mathrm{D}_{0}$ & 73.38 a-d & $4.08 \mathrm{~d}-\mathrm{f}$ & $10.47 \mathrm{a}-\mathrm{d}$ & $15.01 \mathrm{a}-\mathrm{g}$ & $32.70 \mathrm{~cd}$ & $2.18 \mathrm{a}-\mathrm{d}$ & $45.19 \mathrm{c}-\mathrm{e}$ \\
\hline $\mathrm{C}_{1} \mathrm{D}_{1}$ & 70.91 a-f & $3.77 \mathrm{fg}$ & $9.30 e^{-i}$ & $14.23 \mathrm{f}-\mathrm{i}$ & $23.51 \mathrm{ij}$ & $1.65 \mathrm{gh}$ & $40.15 \mathrm{fg}$ \\
\hline $\mathrm{C}_{1} \mathrm{D}_{2}$ & $65.92 \mathrm{f}-\mathrm{h}$ & $3.88 \mathrm{fg}$ & 9.83 b-i & $14.63 \mathrm{c}-\mathrm{h}$ & $27.50 \mathrm{fg}$ & $1.88 \mathrm{~d}-\mathrm{g}$ & $44.05 \mathrm{~d}-\mathrm{f}$ \\
\hline $\mathrm{C}_{1} \mathrm{D}_{3}$ & $62.08 \mathrm{~h}$ & & 9.10 hi & 13.11 i0 & $21.30 \mathrm{j}$ & & $38.04 \mathrm{~g}$ \\
\hline $\mathrm{C}_{2} \mathrm{D}_{0}$ & $72.27 \mathrm{a}-\mathrm{d}$ & $4.58 \mathrm{~d}$ & $10.16 \mathrm{a}-\mathrm{h}$ & 15.24 a-f & $35.62 b$ & $2.34 \mathrm{ab}$ & $37.97 \mathrm{~g}$ \\
\hline $\mathrm{C}_{2} \mathrm{D}_{1}$ & $69.38 \mathrm{~b}-\mathrm{g}$ & 4.02 ef & $9.64 c-i$ & $14.37 e^{-i}$ & $26.49 \mathrm{f}-\mathrm{h}$ & $1.84 e-h$ & $43.75 \mathrm{~d}-\mathrm{f}$ \\
\hline $\mathrm{C}_{2} \mathrm{D}_{2}$ & $66.11 e^{-h}$ & $4.16 \mathrm{~d}-\mathrm{f}$ & 10.03 b-i & $14.86 \mathrm{~b}-\mathrm{g}$ & 30.52 de & $2.05 \mathrm{~b}-\mathrm{f}$ & 45.75 b-e \\
\hline $\mathrm{C}_{2} \mathrm{D}_{3}$ & $64.54 \mathrm{gh}$ & $2.83 \mathrm{ij}$ & $9.43 \mathrm{~d}-\mathrm{i}$ & $13.45 \mathrm{hi}$ & $22.50 \mathrm{ij}$ & $1.67 \mathrm{gh}$ & 43.94 d-f \\
\hline $\mathrm{C}_{3} \mathrm{D}_{0}$ & $74.33 \mathrm{ab}$ & $5.67 \mathrm{a}-\mathrm{c}$ & $10.57 \mathrm{a}-\mathrm{c}$ & $16.22 \mathrm{ab}$ & $36.07 \mathrm{~b}$ & $2.22 \mathrm{a}-\mathrm{c}$ & $49.63 \mathrm{ab}$ \\
\hline $\mathrm{C}_{3} \mathrm{D}_{1}$ & $71.12 \mathrm{a}-\mathrm{f}$ & $4.45 \mathrm{de}$ & $10.06 \mathrm{~b}-\mathrm{i}$ & $15.66 \mathrm{a}-\mathrm{c}$ & 30.54 de & $1.95 \mathrm{c}-\mathrm{g}$ & 45.44 c-e \\
\hline $\mathrm{C}_{3} \mathrm{D}_{2}$ & $68.23 \mathrm{~d}-\mathrm{g}$ & $5.53 \mathrm{bc}$ & 10.40 a-e & $15.9 \mathrm{a}-\mathrm{c}$ & 33.78 bc & 2.12 a-e & $48.41 \mathrm{a}-\mathrm{c}$ \\
\hline $\mathrm{C}_{3} \mathrm{D}_{3}$ & $66.21 e^{-h}$ & $3.06 \mathrm{hi}$ & $9.63 c-i$ & $14.50 \mathrm{~d}-\mathrm{h}$ & $25.06 \mathrm{~g}-\mathrm{i}$ & $1.73 \mathrm{f}-\mathrm{h}$ & 42.82 ef \\
\hline $\mathrm{C}_{4} \mathrm{D}_{0}$ & $75.42 \mathrm{a}$ & $6.16 \mathrm{a}$ & $11.20 \mathrm{a}$ & $16.33 \mathrm{a}$ & $39.13 \mathrm{a}$ & $2.40 \mathrm{a}$ & $51.67 \mathrm{a}$ \\
\hline $\mathrm{C}_{4} \mathrm{D}_{1}$ & $73.68 \mathrm{a}-\mathrm{c}$ & $5.25 \mathrm{c}$ & $10.30 \mathrm{a}-\mathrm{f}$ & 15.76 a-d & 28.70 ef & $1.82 \mathrm{e}-\mathrm{h}$ & $46.99 b-d$ \\
\hline $\mathrm{C}_{4} \mathrm{D}_{2}$ & 71.42 a-e & $5.91 \mathrm{ab}$ & $10.80 \mathrm{ab}$ & $16.10 \mathrm{ab}$ & 34.48 bc & 2.14 a-e & $48.22 \mathrm{a}-\mathrm{c}$ \\
\hline $\mathrm{C}_{4} \mathrm{D}_{3}$ & $68.05 \mathrm{~d}-\mathrm{g}$ & $3.66 \mathrm{fg}$ & $9.27 \mathrm{f}-\mathrm{i}$ & $14.70 \mathrm{c}-\mathrm{h}$ & $27.93 e-g$ & 1.90 e-h & 42.38 ef \\
\hline $\mathrm{SE}$ & 2.65 & 0.27 & 0.55 & 0.67 & 1.43 & 0.17 & 2.00 \\
\hline CV (\%) & 4.71 & 8.07 & 6.78 & 5.58 & 6.18 & 10.87 & 5.62 \\
\hline
\end{tabular}

In a column means having similar letter(s) are statistically similar and those having dissimilar letter(s) differ significantly at $5 \%$ level of significance by DMRT.

Here: $\mathrm{C}_{0}=$ Control (No cowdung), $\mathrm{C}_{1}=25 \%$ less cowdung of recommended dose, $\mathrm{C}_{2}=$ Recommended dose of cowdung, $\mathrm{C}_{3}=25 \%$ higher cowdung of recommended dose and $\mathrm{C}_{4}=50 \%$ higher cowdung of recommended dose; $\mathrm{D}_{0}=$ Control (No water deficit), $\mathrm{D}_{1}=$ Water deficit at crown root initiation stage (20-19 DAS), $\mathrm{D}_{2}=$ Water deficit atbooting stage (45-54 DAS) and $\mathrm{D}_{3}=$ Water deficit at anthesis stage (55-64 DAS). 
Table 6. Interaction effects of different levels of cowdung and water deficit imposition treatment on yield and harvest index of wheat

\begin{tabular}{|c|c|c|c|c|}
\hline Interaction & $\begin{array}{l}\text { Grain yield } \\
\text { plant }^{-1} \\
\text { (g) }\end{array}$ & $\begin{array}{l}\text { Straw yield } \\
\text { plant }^{-1} \\
\text { (g) }\end{array}$ & $\begin{array}{l}\text { Biological } \\
\text { yield plant } \\
\text { (g) }\end{array}$ & $\begin{array}{l}\text { Harvest index } \\
\text { (\%) }\end{array}$ \\
\hline $\mathrm{C}_{0} \mathrm{D}_{0}$ & $4.82 \mathrm{~d}-\mathrm{g}$ & 6.36 a-e & $11.18 \mathrm{c}-\mathrm{f}$ & $43.11 \mathrm{ab}$ \\
\hline $\mathrm{C}_{0} \mathrm{D}_{1}$ & $4.13 \mathrm{~h}-\mathrm{j}$ & $5.70 \mathrm{~d}-\mathrm{f}$ & $9.83 \mathrm{~g}-\mathrm{i}$ & $42.01 \mathrm{ab}$ \\
\hline $\mathrm{C}_{0} \mathrm{D}_{2}$ & $4.91 \mathrm{~d}-\mathrm{f}$ & $6.44 \mathrm{a}-\mathrm{d}$ & $11.35 c-f$ & $43.26 \mathrm{ab}$ \\
\hline $\mathrm{C}_{0} \mathrm{D}_{3}$ & $3.51 \mathrm{k}$ & $5.10 \mathrm{f}$ & $8.61 \mathrm{j}$ & $40.77 \mathrm{~b}$ \\
\hline $\mathrm{C}_{1} \mathrm{D}_{0}$ & $5.13 \mathrm{~b}-\mathrm{d}$ & $6.48 \mathrm{a}-\mathrm{d}$ & 11.61 b-e & $44.19 \mathrm{ab}$ \\
\hline $\mathrm{C}_{1} \mathrm{D}_{1}$ & $4.50 \mathrm{f}-\mathrm{i}$ & $5.97 b-f$ & $10.47 e^{-h}$ & $42.98 \mathrm{ab}$ \\
\hline $\mathrm{C}_{1} \mathrm{D}_{2}$ & $5.07 \mathrm{c}-\mathrm{e}$ & $6.45 \mathrm{a}-\mathrm{d}$ & 11.52 b-e & $44.00 \mathrm{ab}$ \\
\hline $\mathrm{C}_{1} \mathrm{D}_{3}$ & $3.86 \mathrm{jk}$ & 5.40 ef & $9.26 \mathrm{ij}$ & $41.67 \mathrm{~b}$ \\
\hline $\mathrm{C}_{2} \mathrm{D}_{0}$ & $5.45 \mathrm{a}-\mathrm{c}$ & $6.76 \mathrm{a}-\mathrm{c}$ & $12.21 \mathrm{a}-\mathrm{c}$ & $44.64 \mathrm{ab}$ \\
\hline $\mathrm{C}_{2} \mathrm{D}_{1}$ & $4.41 \mathrm{f}-\mathrm{i}$ & $5.88 c-f$ & $10.29 \mathrm{f}-\mathrm{i}$ & $42.86 \mathrm{ab}$ \\
\hline $\mathrm{C}_{2} \mathrm{D}_{2}$ & 5.12 b-e & $6.47 \mathrm{a}-\mathrm{d}$ & 11.59 b-e & $44.18 \mathrm{ab}$ \\
\hline $\mathrm{C}_{2} \mathrm{D}_{3}$ & $4.07 \mathrm{ij}$ & $5.58 \mathrm{~d}-\mathrm{f}$ & 9.65 h-j & $42.18 \mathrm{ab}$ \\
\hline $\mathrm{C}_{3} \mathrm{D}_{0}$ & $5.62 \mathrm{ab}$ & $7.08 \mathrm{a}$ & $12.70 \mathrm{ab}$ & $44.25 \mathrm{ab}$ \\
\hline $\mathrm{C}_{3} \mathrm{D}_{1}$ & $4.62 e^{-h}$ & $6.10 \mathrm{a}-\mathrm{e}$ & $10.72 e^{-h}$ & $43.10 \mathrm{ab}$ \\
\hline $\mathrm{C}_{3} \mathrm{D}_{2}$ & $5.48 \mathrm{a}-\mathrm{c}$ & $7.06 \mathrm{a}$ & $12.54 \mathrm{ab}$ & $43.70 \mathrm{ab}$ \\
\hline $\mathrm{C}_{3} \mathrm{D}_{3}$ & $4.23 \mathrm{~h}-\mathrm{j}$ & $5.74 \mathrm{~d}-\mathrm{f}$ & $9.97 \mathrm{~g}-\mathrm{i}$ & $42.43 \mathrm{ab}$ \\
\hline $\mathrm{C}_{4} \mathrm{D}_{0}$ & $5.93 \mathrm{a}$ & $6.91 \mathrm{ab}$ & $12.84 \mathrm{a}$ & $46.18 \mathrm{a}$ \\
\hline $\mathrm{C}_{4} \mathrm{D}_{1}$ & $4.86 \mathrm{~d}-\mathrm{g}$ & $6.40 \mathrm{a}-\mathrm{d}$ & $11.26 \mathrm{c}-\mathrm{f}$ & $43.16 \mathrm{ab}$ \\
\hline $\mathrm{C}_{4} \mathrm{D}_{2}$ & $5.32 \mathrm{~b}-\mathrm{d}$ & $6.75 \mathrm{a}-\mathrm{c}$ & $12.07 \mathrm{a}-\mathrm{d}$ & $44.08 \mathrm{ab}$ \\
\hline $\mathrm{C}_{4} \mathrm{D}_{3}$ & $4.37 \mathrm{~g}-\mathrm{i}$ & $5.82 \mathrm{c}-\mathrm{f}$ & $10.19 \mathrm{f}-\mathrm{i}$ & $42.89 \mathrm{ab}$ \\
\hline $\mathrm{SE}$ & 0.25 & 0.49 & 0.59 & 2.06 \\
\hline CV (\%) & 6.45 & 9.72 & 6.56 & 5.84 \\
\hline
\end{tabular}

In a column means having similar letter(s) are statistically similar and those having dissimilar letter(s)differ significantly at $5 \%$ level of significance by DMRT.

Here: $C_{0}=$ Control (No cowdung), $C_{1}=25 \%$ less cowdung of recommended dose, $C_{2}=$ Recommended dose of cowdung, $C_{3}=25 \%$ higher cowdung of recommended dose and $C_{4}=50 \%$ higher cowdung of recommended dose; $\mathrm{D}_{0}=$ Control (No water deficit), $\mathrm{D}_{1}=$ Water deficit at crown root initiation stage (20-19 DAS), $\mathrm{D}_{2}=$ Water deficit atbooting stage (45-54 DAS) and $\mathrm{D}_{3}=$ Water deficit at anthesis stage (55-64 DAS).

\section{Conclusion}

It isconcluded from the result that although both of 50\% and 25\% higher cowdung than recommended dose gave the highest yield but 25\% higher cowdung than recommended dose (as it save $25 \%$ cowdung) may be suggested to overcome yield loss due to water deficit conditionat booting stage of wheat $\left(\mathrm{D}_{2}\right)$.

\section{Acknowledgement}

The author acknowledged the Sher-e-Bangla Agricultural University Research System (SAURES) for providing financial support to conduct the study at Sher-e-Bangla Agricultural University campus. 


\section{References}

Akram, M. 2011. Growth and yield components of wheat under water stress of different growth stages. Bangladesh J. Agril. Res. 36(3): 455-468.

Alghabari, F. and M.Z. Ishan. 2018. Effect of drought stress on growth, grain filling duration, yield and quality attributes of barley (Houdium vilgare L.). Bangladesh J. Bot.47(3): 421-428.

Amin, A.K.M.R. and M.A. Baque. 2020. Influence of organic manures on drought stress at different growth stages of Wheat. Bangladesh Agron. J. 23(2): 81-86.

BBS (Bangladesh Bureau of Statistics). 2019. Year Book of Agricultural Statistic-2018. Statistics and informatics Div., Ministry of Planning. Govt. People's Repub., Bangladesh, Dhaka.

BARI (Bangladesh Agricultural Research Institute). 2020. Gom utpadon projukti (In Bangla). Krishi projukti hatboi (part 1), $4^{\text {th }}$ edition. pp.13-14.

Chen, X., D. Min, T.A.Yasir and Y.G. Hu. 2012. Field crops research evaluation of 14 morphological, yield-related and physiological traits as indicators of drought tolerance in Chinese winter bread wheat revealed by analysis of the membership function value of drought tolerance (MFVD). Field Crop Res. 137: 195-201.

Daryanto, S., L. Wang, P.A. Jacinthe, L. Cordain, A. Simopoulos, D. Ray, N.Mueller, P. West, J. Foley and N. Kadam. 2016. Global synthesis of drought effects on maize and wheat production. Hui D, editor. PLOS One. 11: 0156362. https://doi.org/10.1371/journal.pone.0156362

FAO/UNDP (Food and Agriculture Organization). 1988.Land resources appraisal of Bangladesh for Agricultural development report 2: Agroecological regions of Bangladesh. FAO/UNDP.

FAO (Food and Agriculture Organization). 2014.Flood irrigation of wheat on the transitional redbrown earth. I. Effect of duration of ponding on soil water, plant growth, yield and $\mathrm{N}$ uptake. Australian J. Argil. Res. 42(7): 1023-1035.

Geravandi, M., E. Farshadfar and D. Kahrizi. 2011. Evaluation of some physiological traits as indicators of drought tolerance in bread wheat genotypes. Russian J. Plant Physiol. 58: 6975.

Hammad, H.M.,A. Khaliq, A. Ahmad and K. Laghar. 2011. Influence of different organic manures on wheat productivity. Intl. J. Agric. Biol. 13(1):137-140.

Hossain, A., J.A. Teixeira da Silva, M.V. Lozovskaya and V.P. Zvolinsky. 2012. High temperature combined with drought affect rainfed spring wheat and barley in South-Eastern Russia: I. Phenology and growth. Saudi J. Biol. Sci. 19: 473-487.

Ibrahim,M., A. Hussain, M. Iqbal and E. Valeem. 2008. Response of wheat growth and yield to various levels of compost and organic manure. Pakistan J. Bot. 40(5): 2135-2141.

Kilic, H. and Y. Tacettin. 2010. The effect of drought stress on grain yield, yield components and some quality traits of durum wheat (Triticumturgidum sp. durum). Not. Bot. Hort. Agrobot. Cluj-Napoca. 38: 164-170.

Liu, Y., H. Liang, X. Lv, D. Liu, X. Wen and Y. Liao. 2016. Effect of polyamines on the grain filling of wheat under drought stress. Plant Physiol. Biochem. 100: 113-129.

Nezhadahmadi, A., Z.H. Prodhan and G. Faruq. 2013. Drought tolerance in wheat (Review article). The Sci. World J. 2013(1): 1-12.

Nouri-Ganbalani, A., G. Nouri-Ganbalani, and D. Hassanpanah. 2009. Effects of drought stress condition on the yield and yield components of advanced wheat genotypes in Ardabil, Iran. J. Food Agric. Environ. 77: 228-234.

Sarwar, G. 2005. Use of compost for crop production in Pakistan. Ökologie und Umweltsicherung. 26/2005. Universität Kassel. 
Uyanoz, R., U. Cetin and E. Karaarslan. 2006. Effect of organic materials on yields and nutrient accumulation of wheat. J. Plant Nut. 29(5): 959-974.

Waraich, E.A., R. Ahmad and M.Y. Ashraf. 2011. Role of mineral nutrition in alleviation of drought stress in plants. Australian J. Crop Sci. 5: 764-777.

Waraich, E.A., R. Ahmad, S. Saifullah Ahmad and A. Ahmad. 2010. Impact of water and nutrient management on the nutritional quality of wheat. J. Plant Nutr. 33: 640-653.

Yassen, A.A., M. Abd El-Hady and S.M. Zaghloul. 2006. Replacement part of mineral N fertilizer by organic ones and its effect on wheat plant under water regime conditions.World J. Agric. Sci. 2: 421-428. 